\title{
How powerful are integer-valued martingales?
}

\author{
Laurent Bienvenu ${ }^{1}$, Frank Stephan ${ }^{2}$ and Jason Teutsch ${ }^{3}$ \\ 1 LIAFA, CNRS \& Université de Paris 7, France \\ laurent.bienvenu@liafa.jussieu.fr \\ 2 National University of Singapore \\ fstephan@comp.nus.edu.sg \\ ${ }^{3}$ Center for Communications Research-La Jolla, USA \\ jrteuts@ccrwest.org
}

\begin{abstract}
In the theory of algorithmic randomness, one of the central notions is that of computable randomness. An infinite binary sequence $X$ is computably random if no recursive martingale (strategy) can win an infinite amount of money by betting on the values of the bits of $X$. In the classical model, the martingales considered are real-valued, that is, the bets made by the martingale can be arbitrary real numbers. In this paper, we investigate a more restricted model, where only integer-valued martingales are considered, and we study the class of random sequences induced by this model.
\end{abstract}

\section{Gambling with or without coins}

One of the main approaches to define the notion of random sequence is the socalled "unpredictability paradigm". We say that an infinite binary sequence is "random" if there is no effective way to win arbitrarily large amounts of money by betting on the values of its bits. The main notion arising from this paradigm is computable randomness, but other central notions such as Martin-Löf randomness, Schnorr randomness, and Kurtz randomness, can be formulated in this setting. For all of these notions, we consider models of games where the player can, at each turn, bet any amount of money between 0 and his current capital. In "practice" however, one cannot go into a casino and bet arbitrarily small sums of money: there is always a unit value, and any bet made has to be a multiple of this value. Some casinos (and games) also impose upper limits on the amount of capital the one can gamble in each round of play. In the following exposition, we examine the consequences of restricting betting amounts to integers and finite sets.

To formalize the unpredictability paradigm, we need the central notion of martingale. A martingale is a betting strategy for a fair game and is formally represented by a function that corresponds to the gambler's fortune at each moment in time. Let $\{0,1\}^{*}$ denote the set of all finite binary sequences, and $\{0,1\}^{\omega}$ is the set of all countably infinite binary sequences (a.k.a reals). Any 
function $M:\{0,1\}^{*} \rightarrow \mathbb{R}^{+}$which satisfies the fairness condition

$$
M(\sigma)=\frac{M(\sigma 0)+M(\sigma 1)}{2}
$$

for all $\sigma \in\{0,1\}^{*}$ is called a martingale. $M(\sigma)$ corresponds to the gambler's capital after having already bet on the finite sequence $\sigma$. The fairness condition (1.1) says that the amount of money gained from an outcome of " 0 " is the same that would be lost from an outcome of "1". It is important to note that our definition of martingale is a very restricted version of what is usually referred to as "martingale" in probability theory, where it is defined to be a sequence $X_{0}, X_{1}, \ldots$ of real-valued random variables (possibly taking negative values) such that for all $n$

$$
\mathbb{E}\left[X_{n+1} \mid X_{0}, X_{1}, \ldots, X_{n}\right]=X_{n} .
$$

To make the distinction, we call such a sequence a martingale process. A martingale is called recursive if $M$ is a recursive function. Throughout this exposition, "martingale" and "recursive martingale" will be used synonymously.

For any $A \in\{0,1\}^{\omega}, A \| n$ is the finite binary sequence, or initial segment, consisting of the first $n$ digits of $A . \bar{A}$ denotes the complement of a set $A$ (when seen as a subset of $\mathbb{N}$ ). We also identify sets with their characteristic sequences. $|\sigma|$ denotes the length of a binary sequence $\sigma$. A martingale $M$ succeeds on $A \in$ $\{0,1\}^{\omega}$ if $M$ achieves arbitrary sums of money over $A$, that is, $\lim _{\sup _{n}} M(A \|$ $n)=\infty$. Otherwise $A$ defeats $M$. $M$ Schnorr-succeeds on a set $A$ if $M$ succeeds on $A$ and there exists a recursive, non-decreasing, unbounded function $f$ such that $f(n)<M(A \| n)$ for infinitely many $n$. M Kurtz-succeeds on a set $A$ if $M$ succeeds on $A$ and there exists a recursive, non-decreasing, unbounded function $f$ such that $f(n)<M(A \| n)$ for all $n$. We can now define the main classical notions of randomness in terms of martingales.

Definition 1. A sequence $A \in\{0,1\}^{\omega}$ is called computably random if $A$ defeats every martingale. If no martingale Schnorr-succeeds on $A$, then $A$ is Schnorr random. If no martingale Kurtz-succeeds on $A$, then $A$ is Kurtz random (equivalently, $A$ is Kurtz random if and only if $A$ does not belong to any $\Pi_{1}^{0}$ subset of $\{0,1\}^{\omega}$ of measure 0$)$.

In this paper, we shall consider games where the player can only make bets of integer value. For $M$ a martingale and $\sigma \in\{0,1\}^{*},|M(\sigma 0)-M(\sigma)|$ is called the wager at $\sigma$. Now, given a set $V$ of non-negative integers, we say that a martingale is $V$-valued if for all $\sigma$ the wager of $M$ at $\sigma$ belongs to $V$, unless $M$ does not have enough capital in which case the wager at $\sigma$ is 0 . Formally, $M$ is $V$-valued if for all $\sigma \in\{0,1\}^{*}$ and $a \in\{0,1\}, M(\sigma)<\min (V) \Rightarrow M(\sigma a)=M(\sigma)$ and $M(\sigma) \geq \min (V) \Rightarrow|M(\sigma a)-M(\sigma)| \in V$. A martingale whose wagers are integers is called an integer-valued martingale. In case $V$ is finite we say that $M$ is finitely-valued and if $V$ is a singleton, that $M$ is single-valued.

Definition 2. A real $X$ is $V$-valued random if no $V$-valued martingale succeeds on $X$. A real $X$ is a finitely-valued / integer-valued / single-valued random if no finitely-valued / integer-valued / single-valued martingale succeeds on $X$. 
The rest of the paper studies how these new notions of randomness interact with the classical ones. We will prove the implications of the following diagram:

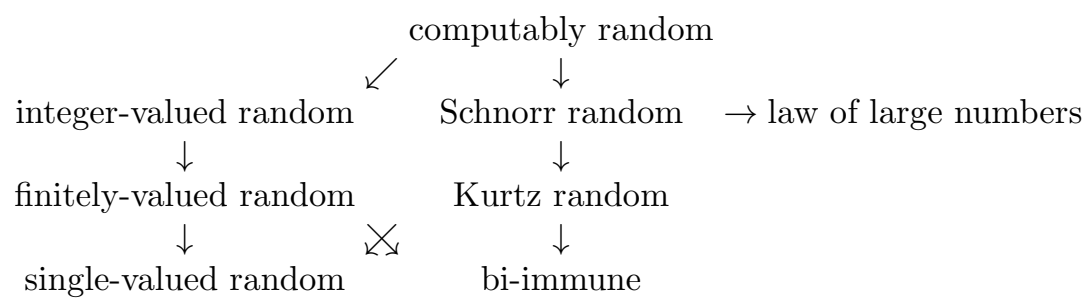

and we shall further see that no other implication than those indicated (and their transitive closure) holds. We than an infinite set is called immune if it contains no infinite r.e. set. Even stronger, an infinite set $A=\left\{a_{0}<a_{1}<a_{2}<\cdots\right\}$ is hyperimmune if there exists no recursive function $f$ such that $f(n)>a_{n}$ for all $n$ 13. A (hyper)immune set whose complement is also (hyper)immune is called bi-(hyper)immune. Thus a member of $\{0,1\}^{\omega}$ is bi-immune if and only if there no recursive list of positions containing all 0's or all 1's. A set $A$ is $\Sigma_{0}^{n}$ if $A$ can be defined using a formula with $n$ quantifiers followed by a recursive predicate where the leading quantifier is existential. $\mathbf{0}^{\prime}$ denotes the halting set, and we say $A$ is $B$-recursive if $A$ is Turing reducible to $B$.

For additional background on algorithmic randomness, see the forthcoming book of Downey and Hirschfeldt [4] and the new book of Nies [9].

If we were to ask someone what the absolute minimum one could expect from a set called "random," you might receive one of the following two responses:

1. The set obeys the law of large numbers.

2. The set is bi-immune.

The person who says "1" believes that a set which does not follow the law of large numbers exhibits a probabilistic bias in its distribution of 0's and 1's. The person who says " 2 " believes that a set with an infinite recursive subset of 0's or 1's yields algorithmic bias. There exists, however, a third possibility:

3. The set is single-valued random.

" 3 " closely matches our intuition in the sense that one should not be able to predict successive outcomes resulting from a "random" process. From a practical point-of-view, single-valued randomness also makes sense. If you have to sit out $2^{1000}$ rounds of roulette before placing a sure bet, as might occur when gambling on a non-bi-immune set, then with probability 1 the casino has already closed while you were waiting for this opportunity. In Section 31 we shall prove that notion " 3 " indeed differs from notions " 1 " and " $2 . "$

The separation of Kurtz randomness and Schnorr randomness is folklore (we will see in a moment how it can be proven). A somewhat more difficult 
result is the separation of computable randomness and Schnorr randomness. The separation of these two notions was proven by Wang who constructed a Schnorr random sequence $X$ together with a martingale $M$ that succeeds on $X$. It turns out that in Wang's construction, the martingale $M$ is already $\{0,1\}$ valued, hence it immediately follows that Schnorr randomness (a fortiori Kurtz randomness) does not imply finitely-valued randomness (and a fortiori integervalued randomness).

Theorem 3 (Wang [15]). There exists a Schnorr random $X \in\{0,1\}^{\omega}$ and a $\{0,1\}$-valued martingale $M$ such that $M$ succeeds on $X$.

In Section 2 we shall see that conversely, integer-valued randomness does not imply Schnorr randomness, and a fortiori computable randomness.

\section{Integer-valued martingales and genericity}

There is an essential difference between rational-valued and integer-valued martingales. The latter can always be permanently defeated while in general the former cannot be. Consider the example of a player starting with an initial capital of 1 who at each turn bets half of its capital on the value 1 (that is, the corresponding martingale $M$ satisfies $M(\sigma 0)=M(\sigma) / 2$ and $M(\sigma 1)=3 M(\sigma) / 2$ for all $\left.\sigma \in\{0,1\}^{*}\right)$. This is a rational-valued martingale with the following property. Pick a stage $s$ of the game; no matter how unlucky the player has been before that stage, she always has a chance to recover. More precisely, for any finite sequence of outcomes $\sigma \in\{0,1\}^{*}$, no matter how small $M(\sigma)$ is, the player can still win the game if the remaining of the outcomes contains a lot of 0 's (for example the player wins against the sequence $\sigma 0000 \ldots$ ). This phenomenon no longer holds for integer-valued martingales, and in fact the opposite is true, that is, no matter how lucky the player has been up to stage $s$, there is always a risk for her to see her strategy permanently defeated at some stage $s^{\prime}>s$. This is expressed by the following lemma.

Lemma 4. Let $M$ be an integer-valued martingale. For any $\sigma \in\{0,1\}^{*}$, there exists an extension $\tau(\sigma, M) \in\{0,1\}^{*}$ of $\sigma$ such that $M\left(\tau^{\prime}\right)=M(\tau(\sigma, M))$ for all extensions $\tau^{\prime}$ of $\tau(\sigma, M)$ (in particular the strategy $M$ does not succeed on any $X \in\{0,1\}^{\omega}$ extending $\left.\tau(\sigma, M)\right)$.

Proof. Let $M, \sigma$ be fixed. We construct the string $\tau=\tau(\sigma, M)$ via the algorithm:

1. Set $\tau \leftarrow \sigma$

2. While there exists an extension $\tau^{\prime}$ of $\tau$ such that $M\left(\tau^{\prime}\right)<M(\tau)$

Choose any such $\tau^{\prime}$ and set $\tau \leftarrow \tau^{\prime}$ (and go back to step 2.)

\section{3. $\operatorname{Return}(\tau)$}

Note that this is algorithm in a general sense, that is, we do not claim that it can be implemented in a computable way (and indeed it cannot be, because 
the condition of the "While" loop needs to check the values $M\left(\tau^{\prime}\right)$ for all extensions of $\tau$ and there are infinitely many of them), but only that it outputs a correct value of $\tau$. First, to see that the algorithm terminates, notice that after each execution of the While loop, the value of $M(\tau)$ is decreased, and because $M$ has integer values, this means that $M(\tau)$ is decreased by at least 1 . Therefore the While loop is executed at most $k=M(\sigma)$ times. We also claim that the output $\tau$ is correct: indeed it must fail the condition of the While loop, that is, for all extensions $\tau^{\prime}$ of $\tau$ one has $M\left(\tau^{\prime}\right) \geq M(\tau)$. But the fairness condition of martingales implies that in that case, $M\left(\tau^{\prime}\right)=M(\tau)$ for all extensions $\tau^{\prime}$ of $\tau$ (this can be checked by a straightforward induction).

From a topological perspective, the above result shows that any integer-valued martingale $M$ is defeated on a dense open set. Indeed, for any $\sigma, M$ is defeated by every sequence $X \in[\tau(\sigma, M)]$ hence $M$ is defeated by any sequence in the dense open set

$$
\mathcal{U}_{M}=\bigcup_{\sigma \in\{0,1\}^{*}}[\tau(\sigma, M)]
$$

(it is dense as for any $\sigma,[\tau(\sigma, M)] \subseteq[\sigma]$ by construction). Therefore, the set of integer-valued random sequences contains the intersection over all integer-valued martingales $\bigcap \mathcal{U}_{M}$. This is a countable intersection of dense open sets, hence the following corollary.

Corollary 5. The set of integer-valued random sequences is co-meager.

This shows that as a notion of randomness, integer-valued randomness is quite weak. Indeed, one of the most basic properties that we can expect from a random sequence $X$ is that it satisfies the law of large numbers, that is, the number of 0 's in $X \| n$ is $n / 2+o(n)$. It is a routine exercise to show that the set of sequences $X$ satisfying the law of large numbers is a meager set (contained in a countable union of closed set with empty interior). Therefore, in the sense of Baire category, most sequences are integer-valued random but do not satisfy the law of large numbers. On the other hand, it is well-known that any Schnorr random sequence must satisfy the law of large numbers [9, which yields a further corollary.

Corollary 6. There exists a sequence $X \in\{0,1\}^{\omega}$ which is integer-valued random but not Schnorr random.

If we now want to compare integer-valued randomness and Kurtz randomness, the above results are insufficient, as the set of Kurtz random sequences is also a co-meager set. We will prove that Kurtz randomness does not imply integer-valued randomness by looking at the classical counterpart of Baire category, namely genericity. Recall that a set $W \subseteq\{0,1\}^{*}$ is dense if the open set $\bigcup_{\sigma \in W}[\sigma]$ is dense or equivalently if for any string $\sigma$ there exists a string in $W$ extending $\sigma$. We say that $X \in\{0,1\}^{\omega}$ is weakly n-generic if $X$ has a prefix in every dense $\Sigma_{n}^{0}$ set. We further say that $X$ is $n$-generic if for any (not necessarily 
dense) $\Sigma_{n}^{0}$ set of strings $W$, either $X$ has a prefix in $W$ or there exists a prefix of $X$ which has no extension in $W$. For all $n \geq 0$ it holds that

$$
\text { weakly }(\mathrm{n}+1) \text {-generic } \Rightarrow \text { n-generic } \Rightarrow \text { weakly n-generic. }
$$

Kurtz showed that weakly 1-genericity is enough to ensure Kurtz randomness.

Proposition 7 (Kurtz [6]). Any weakly 1-generic sequence $X \in\{0,1\}^{\omega}$ is Kurtz random.

The next two theorems show that more genericity is needed to ensure integervalued randomness. That is, weak 2 -genericity is sufficient, but 1-genericity is not.

Theorem 8. Let $X \in\{0,1\}^{\omega}$ be any weakly 2-generic sequence. Then $X$ is integer-valued random.

Proof. We have shown in Lemma 4 that for any martingale $M \in \mathfrak{D}$, the set of strings

$$
W_{M}=\left\{\sigma: M\left(\sigma^{\prime}\right)=M(\sigma) \text { for all extensions } \sigma^{\prime} \text { of } \sigma\right\}
$$

is dense. It is also easy to see that this set is recursive in $\mathbf{0}^{\prime}$, in particular $W_{M}$ is $\Sigma_{2}^{0}$. By definition, a weak-2-generic sequence $X$ must have a prefix in $W_{M}$ for all integer-valued martingales $M$, and it is clear that if $X$ has a prefix in $W_{M}$, $M$ does not succeed on $X$.

Theorem 9. There exists a 1 -generic sequence $X \in\{0,1\}^{\omega}$ and a $\{0,1\}$-valued martingale $M$ such that $M$ succeeds on $X$.

Proof. We will build the sequence $X$ by constructing an increasing (for the prefix order) sequence $\left(\gamma_{n}\right)$ of strings, then taking $X$ to be the unique element of $\{0,1\}^{\omega}$ having all of the $\gamma_{n}$ as prefixes. The martingale we construct will be $\{0,2\}$-valued, however a successful $\{0,1\}$-valued martingale can also be achieved by cutting the $\{0,2\}$-valued wagers in half.

Let $\left(W_{e}\right)_{e \in \mathbb{N}}$ be an effective enumeration of all $\Sigma_{1}^{0}$ sets of strings. For all $e$, set

$$
F_{e}=\left\{\tau: \exists \sigma \in W_{e,|\tau|} \text { and } \tau \text { extends } \sigma\right\}
$$

and $F_{e}^{\text {min }}$ the set of minimal elements of $F_{e}$, that is, the set of $\tau$ such that $\tau \in F_{e}$ and no strict prefix of $\tau$ is in $F_{e}$. Note that whenever a string $\sigma$ is in $F_{e}$, then so are all strings that extend $\sigma$, and whenever a string $\sigma$ is $F_{e}^{\min }$, then no strict extension of $\sigma$ is. It is clear that the $F_{e}$ and $F_{e}^{\min }$ are (uniformly) recursive sets, and also easy to see that a sequence $Y \in\{0,1\}^{\omega}$ is 1-generic if and only if for all $e$, either $Y$ has a prefix in $F_{e}\left(\right.$ resp. $\left.F_{e}^{\min }\right)$ or some prefix of $Y$ has no extension in $F_{e}$.

We start by defining the martingale $M$ which will succeed on the sequence $X$. It is defined by $M(\epsilon)=12$ and for all $\sigma \in\{0,1\}^{*}$ and $a \in\{0,1\}$ :

$$
M(\sigma a)= \begin{cases}M(\sigma)+2 & \text { if } M(\sigma) \geq \operatorname{permit}(\sigma) \text { and } a=1 \\ M(\sigma)-2 & \text { if } M(\sigma) \geq \operatorname{permit}(\sigma) \text { and } a=0 \\ M(\sigma) & \text { if } M(\sigma)<\operatorname{permit}(\sigma)\end{cases}
$$


where the function permit is defined inductively by

$$
\begin{aligned}
\operatorname{permit}(\epsilon) & =4 ; \\
\operatorname{permit}(\sigma a) & =\min \left[\{\operatorname{permit}(\sigma)+1\} \cup\left\{4 e+4: \sigma a \in F_{e}^{\min }\right\}\right] .
\end{aligned}
$$

for $\sigma \in\{0,1\}^{*}$ and $a \in\{0,1\}$. It is also easy to see that permit and $M$ are recursive, and $M$ is integer-valued (its values are positive because permit $(\sigma) \geq 4$ for all $\sigma$, hence $M$ is never allowed to make a bet if its capital is less than 4).

We now define the sequence of strings $\left(\gamma_{n}\right)$, and an auxiliary sequence $\left(\zeta_{n}\right)$ by setting $\gamma_{0}=\epsilon, \zeta_{0}=\epsilon$ and inductively, for all $n$ :

(a) if there exists an extension of $\gamma_{n}$ in $F_{n}$ then let $\zeta_{n+1}$ be a shortest such extension (chosen effectively), and

(b) if there exists no such extension, let $\zeta_{n+1}=\gamma_{n}$.

Finally, define $\gamma_{n+1}=\zeta_{n+1} 11111111$.

Note that $\zeta_{n}$ can be determined from $\gamma_{n}$ using the oracle $\mathbf{0}^{\prime}$, hence the sequence $X$ obtained in this construction (by taking the limit of the $\gamma_{n}$, or equivalently the limit of the $\zeta_{n}$ ) is also recursive in $\mathbf{0}^{\prime}$. We now prove that $X$ is as wanted by a series of claims.

(i) $X$ is 1-generic. Indeed, at stage $n$ of the construction, either $\zeta_{n}$ is in $F_{n}$ (in fact in $F_{n}^{\mathrm{min}}$ ) or no extension of $\zeta_{n}$ is in $F_{n}$.

(ii) In both cases (a) and (b) of the construction, we ensure that no strict extension of $\zeta_{n}$ is in $F_{n}^{\min }$. Indeed either case (a) holds, and $\zeta_{n}$ is itself in $F_{n}^{\min }$ in which case no strict extension of $\zeta_{n}$ is, or case (b) holds, in which case no extension of $\zeta_{n}$ is in $F_{n}$, and fortiori no extension is in $F_{n}^{\min }$. Additionally, for all $n, \zeta_{n}$ is a strict extension of all $\zeta_{k}$ for $k<n$, therefore we conclude by induction that for all $n$ and all $k \leq n$, no strict extension of $\zeta_{n}$ is in $F_{k}^{\min }$.

(iii) For all $n$, permit $\left(\zeta_{n}\right) \geq 4 n+4$, and moreover, any string $\sigma$ extending $\zeta_{n}$ by at least 4 bits satisfies permit $(\sigma) \geq 4 n+8$. This is shown by induction. First, this holds for $n=0$ : all values of the function permit are greater or equal to 4 , in particular, permit $\left(\zeta_{0}\right) \geq 4$. Now suppose that permit $\left(\zeta_{n}\right) \geq 4 n+4$ for some $n$. As we have seen in claim (ii) above, no strict extension of $\zeta_{n}$ is in $F_{k}$ for any $k \leq n$. Thus, for any extension $\zeta^{\prime}$ of $\zeta_{n}$ and $a \in\{0,1\}$ we have by definition of permit: permit $\left(\zeta^{\prime} a\right) \geq \min \left\{\operatorname{permit}\left(\zeta^{\prime}\right)+1,4 n+8\right\}$. From this, we see by a straightforward induction that string $\sigma$ extending $\zeta_{n}$ by 4 bits or more satisfies permit $(\sigma) \geq 4 n+8$. In particular, $\zeta_{n+1}$ extends $\zeta_{n}$ by at least 8 bits, hence permit $\left(\zeta_{n+1}\right) \geq 4 n+8$, which concludes the induction.

(iv) Similarly, for all $n$, permit $\left(\gamma_{n}\right) \geq 4 n+4$. This is true for $n=0$, and for $n>0$, since $\gamma_{n}$ is an extension of $\zeta_{n-1}$ by 8 bits, it follows from (iii) that $\operatorname{permit}\left(\gamma_{n}\right) \geq 4(n-1)+8=4 n+4$. 
(v) For all $n, M\left(\gamma_{n}\right) \geq \operatorname{permit}\left(\gamma_{n}\right)+8$. This is true for $n=0$. For the induction step, we need to distinguish two cases depending on how $\gamma_{n+1}$ was constructed from $\gamma_{n}$. If we are in the above case (b), then $\gamma_{n+1}=$ $\gamma_{n} 11111111$, and since $M\left(\gamma_{n}\right)>$ permit $\left(\gamma_{n}\right), M$ bets and wins 8 consecutive times, and thus $M\left(\gamma_{n+1}\right)=M\left(\gamma_{n}\right)+16$. Also, in that case permit $\left(\gamma_{n+1}\right) \leq$ permit $\left(\gamma_{n}\right)+8$ by definition of permit (adding one bit to a string can only increase the value of permit by 1 ). From these two facts we can conclude that $M\left(\gamma_{n+1}\right) \geq \operatorname{permit}\left(\gamma_{n+1}\right)+8$. Suppose now that $\gamma_{n+1}$ was constructed according to case (a) above. In that case, we need to precisely analyze the behavior of $M$ and permit between $\zeta_{n}$ and $\gamma_{n}$, i.e. on strings of type $\zeta_{n} \eta$ with $0 \leq|\eta| \leq\left|\gamma_{n}\right|-\left|\zeta_{n}\right|$. First, $\zeta_{n}$ is an extension of $\gamma_{n-1}$ hence by (ii) no string $\zeta_{n} \eta$ is in $F_{k}^{\min }$ for $k<n$. Additionally, since $\gamma_{n}$ belongs to $F_{n}^{\min }$, no prefix of $\gamma_{n}$ does. This shows by definition of permit that for $|\eta| \leq 4$ one has $\operatorname{permit}\left(\zeta_{n} \eta\right)=\operatorname{permit}\left(\zeta_{n}\right)+|\eta|$ and for $|\eta| \geq 4$ one has permit $\left(\zeta_{n} \eta\right) \geq 4 n+8$. On the other hand, $M\left(\gamma_{n}\right) \geq \operatorname{permit}\left(\gamma_{n}\right)+8 \geq 4 n+12$ (by (iv)). Since $M$ can only decrease by 2 at each move, we have $M\left(\zeta_{n} \eta\right) \geq 4 n+6$ for any $|\eta| \leq 3$. But if $|\eta| \geq 4$, as we just saw, the value of permit $\left(\zeta_{n} \eta\right)$ is at least $4 n+8$, and the martingale $M$ is never allowed to bet if its capital is below permit. Hence, it follows that $M\left(\zeta_{n} \eta\right) \geq 4 n+6$ whenever $0 \leq|\eta| \leq\left|\gamma_{n}\right|-\left|\zeta_{n}\right|$. In particular, $M\left(\zeta_{n}\right) \geq 4 n+6$, and since we are in case (a), permit $\left(\zeta_{n}\right) \leq 4 n+4$, thus $M$ is allowed to bet and wins 8 times consecutively, and $M\left(\gamma_{n+1}\right) \geq 4 n+22$. Finally, we have permit $\left(\gamma_{n+1}\right) \leq \operatorname{permit}\left(\zeta_{n}\right)+8 \leq 4 n+12$. This finishes the induction.

We have seen in (i) that $X$ is 1-generic, and from (iv) and (v), it follows that $\lim \sup _{n} M\left(\gamma_{n}\right)=+\infty$, hence $M$ succeeds on $X$.

The sequence $X$ constructed in this last proof is 1-generic, hence by Kurtz's result mentioned above $X$ is also Kurtz random. We therefore get the immediate corollary.

Corollary 10. There exists a sequence $X \in\{0,1\}^{\omega}$ which is Kurtz random but not integer-valued random.

The converse of this result is also true, that is there exists a sequence $X$ which is integer-valued random but not Kurtz random. To prove this, we will need a different approach, via measure-theoretic arguments, which we will present in Section 4 .

Strictly speaking, integer-valued martingales not only impose a lower limit on betting amounts but also require that all wagers be a multiple of the minimum bet. We are therefore left with a question regarding the robustness of integervalued randomness: if we remove the requirement that wagers must be a multiple of the minimum bet, do we still obtain the same notion of randomness?

Open question 11. Let $V$ be the set of all computable reals greater than or equal to 1 unioned with $\{0\}$. Is $V$-valued random the same as integer-valued random? 


\section{$3 \quad$ Finitely-valued martingales}

We now consider the effects of imposing betting limits on martingale strategies. First we separate integer-valued randomness from finitely-valued randomness.

Theorem 12. There exists an integer-valued martingale which succeeds on a finitely-valued random.

Proof. Partition the natural numbers into finite intervals, with $2^{n}$ intervals of length $n$ followed by $2^{n+1}$ intervals of length $n+1$ for every $n$. In a picture:

$$
I_{1,1} I_{1,2} I_{2,1} \ldots I_{2,2^{2}} I_{3,1} \ldots I_{3,2^{3}} I_{4,1} \ldots I_{4,2^{4}} I_{5,1} \ldots
$$

where each interval $I_{n}$, has length $n$. Consider the class of all sets $\mathcal{A}$ which guarantees that at least one "1" lies in each of these intervals. An integer-valued martingale can succeeds on any set in this class by using the "classic" martingale strategy: in each interval bet $\$ 1$ on outcome "1", then bet $\$ 2$ on outcome "1", then bet $\$ 4$ on outcome " 1 ", etc. until the bet is successful and then stop betting until the next interval. In this way, the gambler nets $\$ 1$ income over each interval. After doing this for each of the $2^{n}$ intervals of length $n$, she has enough money to continue this strategy on the next intervals of length $n+1$. Therefore some integer-valued martingale succeeds on every member of $\mathcal{A}$.

On the other hand, we now find a $B \in \mathcal{A}$ on which no finitely-valued martingale succeeds. Let $M_{0}, M_{1}, M_{2}, \ldots$ be a list of all finitely-valued martingales. Let $B(0)=1$. For induction assume $B$ has been defined up through $I_{n}$, and try to define $B$ on $I_{n+1}$ so that

- for some $e, M_{e}$ loses some money over $I_{n+1}$, and

- for every $j<e, M_{j}$ gains no money over $I_{n+1}$.

If all the intervals $B$ are chosen so as to satisfy these requirements, then all finitely-valued martingales will be obliterated. Indeed each index can only be chosen finitely many times to play the role of $e$ before all the capital of $M_{e}$ is destroyed, and therefore the choice of $e$ must go to infinity.

While it is impossible to choose values for $B$ so that these requirements are satisfied on every interval, we can satisfy them often enough to defeat every finitely-valued martingale. Assuming that $I_{n-1}$ has been built, we describe how to build $I_{n}$. Recall that a finitely-valued martingale always wagers integer dollar amounts. For each finitely-valued martingale $M$, let $\max (M)$ denote the maximum possible bet for $M$, and let

$$
L(e)=\sum_{j \leq e}\left[\max \left(M_{j}\right)+1\right] .
$$

Claim: Values for $B$ can be chosen in $I_{n}$ so that $M_{e}$ loses money if she makes a nonzero wager before the last $L(e)$ positions of the interval and is the lowest-indexed martingale to do so. Furthermore for all $j<e, M_{j}$ does not gain any money over $I_{n}$ with these values for $B$. 
Thus if $M_{0}$ bets before the last $L(0)$ positions of $I_{n}, B$ can force $M_{0}$ to lose money, thereby satisfying the construction requirements. So we need only consider the case where $M_{0}$ bets no money before the last $L(0)$ positions of $I_{n}$. By applying the claim above inductively, we may assume that

- each successive $M_{e}$ bets no money prior to the last $L(e)$ positions of $I_{n}$, and

- for each $j \leq e, M_{j}$ earns no profit over $I_{n}$.

Eventually $B$ must encounter some martingale $M_{s}$ which is stupid enough to bet money at the beginning of the interval, at which point the requirements for $I_{n}$ can be satisfied (assuming $I_{n}$ is sufficiently long to have such a "beginning.") If $I_{n}$ is not longer than $L(s)$ then the requirements are not satisfied on $I_{n}$. But we do not worry about this failure because for all $e$ such that $L(e)<\left|I_{n}\right|$, the way of choosing intervals prevents $M_{e}$ from ever earning money again on any interval $I_{n+k}(k \geq 0)$. Thus for every $e$, there is a sufficiently large $N$ so that for all $n>N$, e gains no money from betting on $I_{n}$. So $B$ defeats all finitely-valued martingales.

It remains to prove the claim. We argue by induction. Suppose that $M_{0}$ makes a nonzero wager prior to the last $L(0)$ positions of the interval $I_{n}$, say at position $x_{0}$. We show how $B$ can force $M(0)$ to lose money over $I_{n}$. $B$ can act adversarially throughout the interval except for the constraint inherited from the class $\mathcal{A}$. It follows that $M_{0}$ 's betting amounts must be nondecreasing from position $x_{0}$ until the end of the interval. If not, then $B$ can spend its obligatory " 1 " at the position where $M_{0}$ decreased her bets. $M_{0}$ already has a net loss at this point of decrease, and $B$ can continue to act adversarially until the end. Therefore a decrease in betting amounts after $x_{0}$ would cause $M_{0}$ to lose. Hence $M_{0}$ is forced to bet at least $\$ 1$ each of $L(0)$ times. By the final bet in $I_{n}, M_{0}$ is already behind by at least $\max \left(M_{0}\right)+1$, so this bet is irrelevant; $M_{0}$ has already lost.

Since we have already proved the claim when $M_{0}$ bets before the last $L(0)$ positions, we can now focus on the case where $M_{0}$ bets only during the last $L(0)$ positions. Now it is easy to prevent $M_{0}$ from winning any money: $B$ places a " 1 " anywhere before the $\left|I_{n}\right|-L(0)$ position and then $B$ can act adversarially on the last $L(0)$ positions. Any nonzero wager from $M_{0}$ will now instantly result in a loss for $M_{0}$ because $B$ is free to everywhere disagree with $M_{0}$. Hence it suffices to consider the case where $M_{0}$ does not bet anywhere and $B$ is obligated to post a "1" somewhere before the last $\left|I_{n}\right|-L(0)$ positions.

Curiously, $M_{1}$ now finds herself in exactly the same situation that $M_{0}$ started with. By same argument as above, $B$ can force $M_{1}$ to lose money if $M_{1}$ bets prior to $\left|I_{n}\right|-L(1)$. Therefore we can reduce to the case where $M_{1}$ never bets and $B$ is obligated to provide a "1" somewhere before $L(2)$. The same argument holds for $M_{2}, M_{3}, \ldots$ Eventually some martingale $M_{e}$ has money and is stupid enough to bet before $L(e)$. At this point, the claim is proved.

Remark. $B$ is $\mathbf{0}^{\prime}$-recursive in the above construction. 
Schnorr showed that for any set $A$, a real-valued martingale succeeds on $A$ if and only if a rational-valued martingale succeeds on $A$ (see [11, or [9] p.270). His proof, however, does not carry over to the finitely-valued case.

Open question 13. If we allow finitely-valued martingales to bet real values instead of rationals, do we get the same class of finitely-valued randoms?

\subsection{On single-valued randoms}

For the following discussion, it is useful to keep in mind that a real is single-valued random if and only if it is $\{1\}$-valued random; the particular dollar amount which is bet each round is immaterial. For comparison with Kurtz randomness, we appeal directly to a theorem of Doob ( $\underline{3}$ p.324). The following version for "non-negative" martingales appears in Ross's book ([10, p.316).

Theorem 14. For every martingale $M$, the set of reals on which $M$ succeeds has measure zero. Furthermore, the capital of $M$ converges to some finite value with probability 1.

Later, in Lemma 31, we shall appeal to a more general version of Theorem 14 (see Billingsley [2] p.468). A supermartingale process a sequence $X_{0}, X_{1}, \ldots$ of real-valued random variables (possibly taking negative values) such that for all $n$

$$
\mathbb{E}\left[X_{n+1} \mid X_{0}, X_{1}, \ldots, X_{n}\right] \leq X_{n} .
$$

Theorem 15 (Doob's Martingale Convergence Theorem). Let $X_{0}, X_{1}, \ldots$ be a supermartingale process (where each $X_{i}$ is a random variable). If for some $m \in \mathbb{R}$, we have $X_{n} \geq m$ for all $n$, then almost surely $\lim _{n \rightarrow \infty} X_{n}$ exists and is finite.

Proposition 16. Every Kurtz random is single-valued random.

Proof. Suppose that some single-valued martingale $M$ succeeds on a real $X$. Let $\mathcal{F}$ denote the set of reals on which $M$ converges to some finite value. Then $X$ does not belong to $\mathcal{F}$, and $\mathcal{F}$ has measure one by Theorem 14 . Hence $X$ belongs to the measure zero set $\overline{\mathcal{F}}$.

By definition of single-valued, $M$ is required to bet at every position of the input real. Hence the only way for $M$ to converge to a finite value is to reach the value 0 and become constant. Therefore $\mathcal{F}$ is, in fact, the set of reals on which $M$ eventually goes broke. Thus $\overline{\mathcal{F}}$ is the set of all infinite paths through the tree $\{\sigma: M(\sigma)>0\}$. It follows that $\overline{\mathcal{F}}$ is a recursive $\Pi_{1}^{0}$ class. In summary, $X$ belongs to a recursive $\Pi_{1}^{0}$ class of measure zero and therefore is not Kurtz random.

Remark. The above argument shows even more: every Kurtz random is $V$-valued random for any positive set of integers $V$. 
As promised in the introduction, we now separate the incomparable notions of bi-immunity, single-valued random, and law of large numbers. The reals satisfying the law of large numbers and the bi-immune reals are already known to be different. Their separation can be deduced from the argument given in Proposition 20 with "bi-immune" substituted for "single-valued random."

Theorem 17. There exists a single-valued random which is neither immune nor co-immune.

Proof. Let $M_{0}, M_{1}, \ldots$ be a list of all possible $\{1\}$-computable "gamblers," namely a list of pairs consisting of martingales and their respective initial capital. Define a set $A$ such that

$-A(n)=1$ if $n \equiv 0 \bmod 6$, and

$-A(n)=0$ if $n \equiv 3 \bmod 6$.

The remaining values of $A$ work adversarially against the $M_{i}$ 's. Since the gamblers must bet exactly $\$ 1$ on each value of $A$, the remaining values of $A$ can be chosen so as to force any particular gambler to decrease his capital by a dollar over the course of any three consecutive rounds of play. We define the first initial segment of $A$ so as to exhaust the capital of $M_{0}$, the following interval of $A$ so as to exhaust the capital of $M_{1}$, etc. Since each gambler has only finite capital at any moment, each gambler's capital is exhausted after a finite period of time. Therefore no $\{1\}$-valued martingale succeeds on $A$. Furthermore, by the values assigned at multiples of $3, A$ contains an infinite recursive set as does its complement.

Theorem 18. There exists a $\mathbf{0}^{\prime}$-recursive, bi-hyperimmune set which is not single-valued random.

Proof. Let $\varphi_{0}, \varphi_{1}, \varphi_{2}, \ldots$ be a list of the partial recursive functions. Define a $\mathbf{0}^{\prime}$-recursive function $f$ satisfying

$$
(\forall e<n)\left[\varphi_{e}(f(n)) \downarrow \Longrightarrow f(n+1)>\varphi_{e}(f(n))+2\right]
$$

and let

$$
A=\{x:(\exists n)[f(2 n) \leq x<f(2 n+1)]\} .
$$

Now $A=\left\{a_{0}, a_{1}, a_{2}, \ldots\right\}$ is bi-hyperimmune because for any recursive $\varphi_{k}$,

$$
\varphi_{k}[f(2 k+1)]<f(2 k+2)-2 \leq a_{f(2 k+1)}-1,
$$

and a similar inequality holds for the complement of $A$. On the other hand, the single-valued martingale strategy which bets on $A(n+1)$ what the gambler saw at $A(n)$ will succeed on $A$. This strategy indeed succeeds because each time $A(n+1)$ disagrees with $A(n)$, we have that $A(n+2)$ and $A(n+3)$ agree with $A(n+1)$. So over each three consecutive rounds of betting, the gambler increases his capital by at least $\$ 1$.

Proposition 16 and Theorem 18 together give: 
Corollary 19. There exists a bi-immune set which is not Kurtz random.

Proposition 20. Single-valued random does not imply the law of large numbers and vice-versa.

Proof. Unlike the set of reals which satisfy the law of large numbers, the set of single-valued randoms is co-meager (by Corollary 5 ). Moreover, the real $.010101010 \ldots$ satisfies the law of large numbers but is not single-valued random.

Finally, we note that it is possible to separate single-valued randomness from finite-valued randomness using an argument along the lines of Proposition 17.

Proposition 21. There exists a $\{1,2\}$-valued martingale which succeeds on a single-valued random.

Proof. Similar to Theorem 17 we partition the natural numbers into intervals of length 5 . For the first two numbers $n$ in each interval (that is, $n$ congruent to 0 or $1(\bmod 5))$, set $A(n)=0$ so that gambler can win at these places. The last three spots in each interval are adversarial against the single-valued martingales. $A$ will be able to defeat the $\{1\}$-valued martingale since the best single-valued martingale strategy would first gain $\$ 2$ and then lose $\$ 3$ on each interval, for a net loss of $\$ 1$ per interval. Eventually the single-valued martingale will run out of money. On the other hand, there exists a $\{1,2\}$-valued martingale which always bets $\$ 2$ on each of the first two numbers and $\$ 1$ on the last three numbers in each interval, for a net gain of at least $\$ 4-\$ 3=\$ 1$ per interval (regardless of any adversarial action that may occur in the last 3 places). Thus the money for this $\{1,2\}$-valued martingale on $A$ goes to infinity.

\subsection{On $\{0,1\}$-valued randoms}

We can also separate single-valued randomness from finite-valued randomness.

Proposition 22. Let $V$ be any set containing 0 and at least one other number $n$. Then any $V$-valued random is bi-immune.

Proof. Let $A$ be a set which is not bi-immune; without loss of generality assume that $A$ contains an infinite recursive set $\mathrm{B}$. Then a $V$-valued martingale strategy which bets $n$ dollars on members of $B$ and 0 on $A-B$ will succeed on $A$.

The following corollary is a consequence of the definition of finitely-valued random and Proposition 22.

Corollary 23. finitely-valued random $\Longrightarrow\{0,1\}$-valued random $\Longrightarrow$ biimmune.

Since single-valued random does not imply bi-immune (Theorem 17), we obtain from Corollary 23,

Corollary 24. There exists a $\{0,1\}$-valued random which is not single-valued random. 
Although we were able to separate single-valued randomness from $\{1,2\}$-valued randomness (Proposition 21), the comparison between $\{0,1\}$-valued randoms and $\{0,1,2\}$-valued randoms seems less clear. We leave the reader with the following interesting question.

Open question 25. Is $\{0,1\}$-valued random the same as finitely-valued random?

\section{Integer-valued martingales and Bernoulli measures}

In this last section, we present a proof of the fact that integer-valued randomness does not imply Kurtz randomness. We will get a counter example by choosing a sequence $X$ at random with respect to some carefully-chosen probability measure.

Intuitively speaking, the Lebesgue measure $\lambda$ on the space $\{0,1\}^{\omega}$ corresponds to the random trial where all bits are obtained by independent tosses of a balanced 0/1-coin. An interesting generalization of Lebesgue measure is the class of Bernoulli measures, where for a given parameter $\delta \in[-1 / 2,1 / 2]$ we construct a sequence $X$ by independent tosses of a coin with bias $\delta$ (that is, the coin gives 1 with probability $1 / 2+\delta$ and 0 with probability $1 / 2-\delta$. This can be further generalized by considering an infinite sequence of independent coin tosses where the $n^{\text {th }}$ coin tossed has bias $\delta_{n}$. This leads to the notion of generalized Bernoulli measures. Formally, on the space $\{0,1\}^{\omega}$, given a sequence $\left(\delta_{n}\right)_{n \in \mathbb{N}}$ of numbers in $[-1 / 2,1 / 2]$, a generalized Bernoulli measure of parameter $\left(\delta_{n}\right)_{n \in \mathbb{N}}$ is the unique measure $\mu$ such that for all $\sigma \in\{0,1\}^{*}$ :

$$
\mu([\sigma])=\prod_{n: \sigma(n)=0}\left(1-p_{n}\right) \prod_{n: \sigma(n)=1} p_{n}
$$

where $p_{n}=1 / 2+\delta_{n}$. One can expect that if the $\delta_{n}$ are very small (that is, $\delta_{n}$ tends to 0 quickly), then the generalized Bernoulli measure of parameter $\left(\delta_{n}\right)_{n \in \mathbb{N}}$ will not differ much from Lebesgue measure. This was made precise by Kakutani.

Theorem 26 (Kakutani [5]). Let $\mu$ be the generalized Bernoulli measure of parameter $\left(\delta_{n}\right)_{n \in \mathbb{N}}$. If the condition

$$
\sum_{n \in \mathbb{N}} \delta_{n}^{2}<\infty
$$

holds, then $\mu$ is equivalent to Lebesgue measure $\lambda$, that is, for any subset $\mathcal{X}$ of $\{0,1\}^{\omega}, \mu(\mathcal{X})=0$ if and only if $\lambda(\mathcal{X})=0$. If condition (4.1) does not hold, then $\mu$ and $\lambda$ are inconsistent, that is, there exists some $\mathcal{Y}$ such that $\mu(\mathcal{Y})=0$ while $\lambda(\mathcal{Y})=1$.

If we want to work in a computability setting, we need to consider computable generalized Bernoulli measures, that is, those for which the parameter $\left(\delta_{n}\right)_{n \in \mathbb{N}}$ is a recursive sequence of reals. Vovk [14 showed a constructive analogue of 
Kakutani's theorem for computable generalized Bernoulli measures in relation with Martin-Löf randomness (perhaps the most famous effective notion of randomness, but we do not need it in this paper). The Kakutani-Vovk result has been used many times in the literature $1 / 7 / 8 / 12$. In particular, Bienvenu and Merkle proved the following.

Theorem 27 (Bienvenu and Merkle [1]). Let $\mu$ be a computable generalized Bernoulli measure of parameter $\left(\delta_{n}\right)_{n \in \mathbb{N}}$. If $\sum_{n} \delta_{n}^{2}=+\infty$, then the class of Kurtz random sequences has $\mu$-measure 0 .

To prove that integer-valued randomness does not imply Kurtz randomness, we will construct a computable generalized Bernoulli measure $\mu$ whose parameter $\left(\delta_{n}\right)_{n \in \mathbb{N}}$ converges to 0 sufficiently slowly to have $\sum_{n} \delta_{n}^{2}=+\infty$ (hence by the above $\mu$-almost all sequences $X$ are not Kurtz random, which we will make even more precise) but sufficiently quickly to make $\mu$ close to Lebesgue measure and ensure that $\mu$-almost all sequences are integer-valued random.

Theorem 28. There exists a sequence $X \in\{0,1\}^{\omega}$ which is integer-valued random but not Kurtz random.

Proof. We obtain $X$ by choosing a random sequence with respect to the generalized Bernoulli measure of parameter $\left(\delta_{n}\right)$ with

$$
\delta_{n}=\frac{1}{\sqrt{n \ln n}}
$$

for all $n>1$ (the values of $\delta_{0}$ and $\delta_{1}$ can be set arbitrarily). We have $\sum_{i=2}^{n} \delta_{i}^{2} \sim$ $\ln \ln n$ (this because $\int(t \ln t)^{-1} d t=\ln \ln t$, in particular $\sum_{i} \delta_{i}^{2}=+\infty$ ). By Theorem 27, a sequence $X$ chosen at random according to the measure $\mu$ will not be (with probability 1) Kurtz random. We can even exhibit a martingale $M$ which wins against $\mu$-almost all sequences $X$. It is defined by $M(\epsilon)=1$ and for any string $\sigma$ of length $n$ :

$$
M(\sigma 0)=\left(1-2 \delta_{n}\right) M(\sigma) \quad \text { and } \quad M(\sigma 1)=\left(1+2 \delta_{n}\right) M(\sigma) .
$$

This martingale is in fact the optimal martingale: when playing against a sequence $X$ that is chosen at random with respect to a measure $\nu$, the optimal martingale is defined by $M(\sigma)=\nu([\sigma]) / \lambda([\sigma])$. It is optimal in the sense that for any other martingale $M^{\prime}$, we have for $\mu$-almost all $X \in\{0,1\}^{\omega}: M^{\prime}(X \|$ $n)=O\left[M\left(\begin{array}{lll}X & \| n\end{array}\right)\right]$ (see for example [1]). Here, if we take for $\nu$ our generalized Bernoulli measure $\mu$, the optimal martingale is exactly the martingale $M$. By Theorem 27, for $\mu$-almost all $X, X$ is not Kurtz random, that is, there exists a real-valued martingale $M^{\prime}$ and a recursive order $h$ such that $M^{\prime}(X \| n) \geq h(n)$. But by optimality, for any real-valued martingale $M^{\prime}$ and $\mu$-almost all $X, M^{\prime}(X \| n)=O[M(X \| n)]$. Putting all this together, there exists a recursive order $h$ such that $M(X \| n) \geq h(n)$ for all $n$ and $\mu$-almost all $X$.

However (and this will be crucial for the rest of the argument), $M$ succeeds quite slowly on average. 
Lemma 29. Let $r>0$ be a real number. Then for $\mu$-almost all $X \in\{0,1\}^{\omega}$, one has $M(X \| n)=o\left(n^{r}\right)$.

In order to prove this, we now see $X$ as a random variable with distribution $\mu$. We set for all $n$ :

$$
V_{n}=M(X \Uparrow n)
$$

which is a martingale process. Then set

$$
L_{n}=\ln \left(V_{n}\right)
$$

By definition of $M$ we have for all $n$

$$
V_{n+1}= \begin{cases}\left(1+2 \delta_{n}\right) V_{n} & \text { with probability } 1 / 2+\delta_{n} \\ \left(1-2 \delta_{n}\right) V_{n} & \text { with probability } 1 / 2-\delta_{n}\end{cases}
$$

thus

$$
L_{n+1}= \begin{cases}L_{n}+\ln \left(1+2 \delta_{n}\right) & \text { with probability } 1 / 2+\delta_{n} \\ L_{n}+\ln \left(1-2 \delta_{n}\right) & \text { with probability } 1 / 2-\delta_{n}\end{cases}
$$

Setting

$$
e_{n}=\mathbb{E}\left[L_{n+1}-L_{n}\right]=\left(1 / 2+\delta_{n}\right) \ln \left(1+2 \delta_{n}\right)+\left(1 / 2-\delta_{n}\right) \ln \left(1-2 \delta_{n}\right)
$$

(note en passant that $e_{n} \sim 2 \delta_{n}^{2}$ by same method as (4.19)) we see that

$$
L_{n}^{\prime}=L_{n}-\sum_{i=0}^{n-1} e_{i}
$$

is a martingale process. For all $n$ we have $\left|L_{n+1}^{\prime}-L_{n}^{\prime}\right| \leq e_{n}+2 \delta_{n}$ (here we use the fact that $\ln (1+x) \leq x$ for all $x>-1)$. We can thus apply Azuma's Inequality $\left[?, ?\left[10\right.\right.$ to $L_{n}^{\prime}$ : for all integers $n$ and positive real $a$ one has

$$
\mu\left\{L_{n}^{\prime} \geq a\right\} \leq \exp \left(-\frac{a^{2}}{\sum_{i=0}^{n-1}\left(e_{i}+2 \delta_{i}\right)^{2}}\right)
$$

Taking $a=r \ln n$ (for an arbitrarily small real $r>0$ ) in (4.8) we get

$$
\mu\left\{L_{n}^{\prime} \geq r \ln n\right\} \leq \exp \left(-\frac{r^{2}(\ln n)^{2}}{\sum_{i=0}^{n-1}\left(e_{i}+2 \delta_{i}\right)^{2}}\right)
$$

Since $e_{i} \sim 2 \delta_{i}^{2}$, we have $e_{i}=o\left(\delta_{i}\right)$, so

$$
\sum_{i=0}^{n-1}\left(e_{i}+2 \delta_{i}\right)^{2} \sim \sum_{i=0}^{n-1}\left(2 \delta_{i}\right)^{2} \sim 2 \ln \ln n
$$

Thus for any $n$ large enough:

$$
-\frac{r^{2}(\ln n)^{2}}{\sum_{i=0}^{n-1}\left(e_{i}+2 \delta_{i}\right)^{2}} \leq-2 \ln n
$$


Putting (4.9) and (4.11) together, we get

$$
\mu\left\{L_{n}^{\prime} \geq r \ln n\right\} \leq \frac{1}{n^{2}}
$$

for almost all $n$. By the Borel-Cantelli lemma [10], since $\sum_{n} 1 / n^{2}$ converges, with $\mu$-probability 1 the event $\left[L_{n}^{\prime} \geq r \ln n\right]$ happens only finitely often, that is, with probability 1 , for any $r>0$ and almost all $n, L_{n}^{\prime} \leq r \ln n$. Since

$$
L_{n}=L_{n}^{\prime}+\sum_{i=0}^{n-1} e_{i}
$$

and $\sum_{i=0}^{n-1} e_{i} \sim 2 \ln \ln n$, it follows similarly that, with $\mu$ probability 1 , for any $r>0$ and almost all $n, L_{n} \leq r \ln n$. And as $L_{n}=\ln [M(X \| n)]$, all this entails that with $\mu$-probability $1, M(X \| n) \leq n^{r}$ for any $r>0$ and almost all $n$. This proves Lemma 29.

Let $\mathfrak{D}$ denote the class of integer-valued martingales. We now consider a restriction of integer-valued martingales: let $\mathfrak{D}^{\prime}$ be the subset of $\mathfrak{D}$, consisting of the integer-valued martingales $M$ that further satisfy $M(\sigma)<\sqrt{|\sigma|}$ for almost all $\sigma$. The following lemma shows that the martingales in $\mathfrak{D}^{\prime}$ are essentially as powerful as martingales in $\mathfrak{D}$ against sequences $X$ chosen at random according to $\mu$.

Lemma 30. Let $M \in \mathfrak{D}$. For $\mu$-almost all $X$, there exists $M^{\prime} \in \mathfrak{D}^{\prime}$ such that $M^{\prime}(X \| n)=M(X \| n)$ for almost all $n$.

Let $M \in \mathfrak{D}$. By Lemma 29, $M(X \| n)=o(\sqrt{n})$ almost surely. Hence, for $\mu$ almost all $X$, there exists some $n_{0}$ and all $n>n_{0}, M(X \| n) \leq \sqrt{n} / 2$. For such a pair $\left(X, n_{0}\right)$, we call "invalid" all strings $\sigma$ such that there exists a prefix $\tau$ of $\sigma$ such that $|\tau| \geq n_{0}$ and either $M(\tau 0)>\sqrt{|\tau|}$ or $M(\tau 1)>\sqrt{|\tau|}$, and "valid" any string that is not invalid. Now, define the martingale $M^{\prime}$ by $M^{\prime}(\sigma)=M\left(X \| n_{0}\right)$ for all $\sigma$ with $|\sigma| \leq n_{0}$ and for all $\sigma$ with $|\sigma|>n_{0}$, set $M^{\prime}(\sigma)$ to be $M(\tau)$ with $\tau$ the longest prefix of $\sigma$ that is valid. In other words, $M^{\prime}$ is the trimmed version of $M$ that stops betting forever whenever $M$ makes at stage $n>n_{0}$ a bet that gives it a chance to get a capital $>\sqrt{n}$. It is easy to see that $M^{\prime}$ is itself a martingale, integer-valued as $M$ is, and since $M(X \| n) \leq \sqrt{n} / 2$ for all $n>n_{0}$, all prefixes of $X$ are valid, hence $M^{\prime}(X \| n)=M(X \| n)$ for all $n \geq n_{0}$. This proves the lemma.

Finally, we prove that martingales in $\mathfrak{D}^{\prime}$ are almost surely defeated by a $\mu$-random $X$.

Lemma 31. Let $M \in \mathfrak{D}^{\prime}$. For $\mu$-almost all $X, M$ does not succeed on $X$. 
Let $n_{0}$ be such that $M(\sigma) \leq \sqrt{|\sigma|}$ for all $\sigma$ of length $\geq n_{0}$. Again, we see $X$ as a $\mu$-random variable and define $V_{n}$ by

$$
V_{n}=M(X \Uparrow n)
$$

(note that by definition of $\mathfrak{D}^{\prime}$, we have $V_{n} \leq \sqrt{n}$ for all $n \geq n_{0}$ ) and $L_{n}$ by

$$
L_{n}=\ln [M(X \Uparrow n)]
$$

with the convention $\ln (0)=-1$. For all $n$, define also

$$
\rho_{n}=\frac{M(X \| n+1)-M(X \Uparrow n)}{M(X \| n)}
$$

which is the fraction of its capital the martingale $M$ bets on 1 at stage $n$. It can be negative if $M$ bets on 0 and is by convention 1 if $M(X \| n)=0$. Similarly to (4.5), we have for all $n$ :

$$
L_{n+1}= \begin{cases}L_{n}+\ln \left(1+\rho_{n}\right) & \text { with probability } 1 / 2+\delta_{n} \\ L_{n}+\ln \left(1-\rho_{n}\right) & \text { with probability } 1 / 2-\delta_{n}\end{cases}
$$

Thus we have:

$$
\begin{aligned}
\mathbb{E}\left[L_{n+1}-L_{n}\right] & =\left(1 / 2+\delta_{n}\right) \ln \left(1+\rho_{n}\right)+\left(1 / 2-\delta_{n}\right) \ln \left(1-\rho_{n}\right) \\
& =\frac{1}{2} \ln \left(1-\rho_{n}^{2}\right)+\delta_{n} \ln \left(1+\rho_{n}\right)-\delta_{n} \ln \left(1-\rho_{n}\right) \\
& \leq-\frac{\rho_{n}^{2}}{2}+2 \delta_{n} \rho_{n}
\end{aligned}
$$

(for the last inequality, we use again that $\ln (1+x) \leq x$ for all $x \geq-1$, which is true even for $x=-1$ with our convention $\ln (0)=-1)$. Now, observe that $\rho_{n}$ is either 0 , or of the form $\frac{m}{V_{n}}$ for some integer $m$ as $M$ is integer-valued. In the first case $L_{n+1}=L_{n}$ and in the second case, since $V_{n} \leq \sqrt{n}$ for almost all $n$, we have $\left|\rho_{n}\right| \geq 1 / \sqrt{n}$ for almost all $n$, and therefore $\mathbb{E}\left[L_{n+1}-L_{n}\right] \sim-\frac{\rho_{n}^{2}}{2}<0$ as $\delta_{n}=o(1 / \sqrt{n})=o\left(\rho_{n}\right)$. This shows that $L_{n}$ is ultimately a supermartingale, and it is bounded from below by $\ln (0)=-1$. By Doob's Martingale Convergence Theorem $L_{n}$ converges to a finite value $\mu$-almost surely, hence the same is true for $V_{n}=\exp \left(L_{n}\right)$. Therefore $V_{n}$ is $\mu$-almost surely bounded, hence $M$ is $\mu$-almost surely defeated. This finishes the proof of Lemma 31 .

Theorem 28 now easily follows. Take some $X \in\{0,1\}^{\omega}$ at random according to $\mu$. By Lemma 31, $X$ defeats all $M \in \mathfrak{D}^{\prime} \mu$-almost surely, therefore by Lemma 30. $X$ defeats all $M \in \mathfrak{D} \mu$-almost surely. And finally, by definition of $\mu$ and Theorem 27, $X$ is $\mu$-almost surely not Kurtz random. Therefore, $X$ is $\mu$-almost surely as wanted, hence the existence of at least one $X$ as wanted. 


\section{Non-monotonic betting strategies}

A non-monotonic betting strategy is a betting strategy in which the gambler can bet on the bits of a sequence in any order she chooses [7. A set $X$ is Kolmogorov-Loveland random if no recursive non-monotonic betting strategy succeeds on $X$ and Martin-Löf random if no martingale with an increasing, recursive approximation succeeds on $X$. By a theorem of Muchnik, Semenov, and Uspensky [8] every Martin-Löf random is Kolmogorov-Loveland random, however the reverse containment remains a major open question for the field of algorithmic randomness.

In the real-valued martingale case, every set which is computably random relative to $K$ is also Martin-Löf random (unrelativized, follows from martingale definition of ML-random) and hence Kolmogorov-Loveland random. The situation is a bit different for the case of integer-valued martingales. We shall show that no oracle can be given to a integer-valued martingale which will make it as powerful as its non-monotonic counterpart.

Theorem 32. For every oracle $B$ there is a set $A \leq_{T} B^{\prime}$ and a non-monotonic $\{0,1\}$-valued martingale such that the non-monotonic martingale wins on $A$ while every $B$-recursive monotonic martingale fails to win on $A$.

Proof. Let $M_{1}, M_{2}, M_{3}, \ldots$ be a $B^{\prime}$-recursive list of all integer-valued $B$-recursive martingales with the additional property that $M_{m}$ starts with at most $2^{m}$ dollars; note that such a list can be made by following the $m^{\text {th }}$ program as long as that program belongs to an integer-valued martingale with desired properties and to freeze the martingale as constant (always betting 0 ) if at some time the $B$ recursive martingale turns out to be partial or otherwise ill-defined. Note that although the $m^{\text {th }}$ program might not be total, the $M_{m}$ are all total and uniformly $B$-recursive.

The idea is to construct a recursive partition $I_{0}, I_{1}, I_{2}, \ldots$ of intervals such that $I_{n}$ is so long that only the minority of the positions in the interval are used to behave adversarially to $M_{1}, M_{2}, \ldots, M_{n}$ while the majority of the $x$ in the interval satisfies $A(x)=A\left[\min \left(I_{n}\right)\right]$. The basic idea is to select $A$ as follows on $x \in I_{n}$ :

$$
A(x)= \begin{cases}b & \text { if } M_{m} \text { bets a positive value on } 1-b \text { and } \\ & \text { all } M_{k} \text { with } 1 \leq k<m \text { abstain from betting; } \\ & \text { if } x=\min \left(I_{n}\right) \text { and } \\ & M_{1}, M_{2}, \ldots, M_{n} \text { abstain from betting; } \\ A\left[\min \left(I_{n}\right)\right] \quad \text { if } x>\min \left(I_{n}\right) \text { and } & M_{1}, M_{2}, \ldots, M_{n} \text { abstain from betting. }\end{cases}
$$

Note that the length of $I_{n}$ is determined although one does not know anything about the martingales. Let $a_{1}=2^{\min \left(I_{n}\right)+1}$ and inductively $a_{m+1}=$ $2^{\min \left(I_{n}\right)+m+a_{1}+a_{2}+\ldots+a_{m}}$. The idea is that $a_{m}$ stands for the largest value which $M_{m}$ can reach on the interval $I_{n}$; the upper bound is determined by assuming 
that $M_{m}$ - in the worst case - can double its capital whenever the interval $I_{n}$ is not yet reached or that a martingale $M_{k}$ with $k<m$ is betting which is then given priority in the definition of $A$. One can now verify by induction that each $M_{m}$ can bet on $I_{n}$ only at most $a_{m}$ times until it would go broke and therefore there are at most $a_{1}$ places where $M_{1}$ bets and loses 1 out of its capital; furthermore there are at most $a_{2}$ places where $M_{2}$ bets but $M_{1}$ does not bet and on these $M_{2}$ loses 1 out of its capital; there are at most $a_{3}$ places where $M_{3}$ bets inside $I_{n}$ and $M_{1}, M_{2}$ do not bet and on these places $M_{3}$ loses 1 out of its capital. In total there are at most $a_{1}+a_{2}+\ldots+a_{n}$ places on $I_{n}$ where one of the martingales $M_{1}, M_{2}, \ldots, M_{n}$ are betting and therefore by taking $I_{n}$ to have the length $2\left(1+a_{1}+a_{2}+\ldots+a_{n}\right)$ one gets that $A\left[\min \left(I_{n}\right)\right]$ coincides with the majority of the values $A(x)$ with $x \in I_{n}-\left\{\min \left(I_{n}\right)\right\}$.

This property permits to implement a non-monotonous recursive betting strategy which for every interval $I_{n}$ first reads all the values $A(x)$ with $x \in$ $I_{n}-\left\{\min \left(I_{n}\right)\right\}$ without betting any money on these values and then bets 1 according to the majority of the bits read before on the value $A\left[\min \left(I_{n}\right)\right]$; this bet is correct and a sure win. Hence $A$ can be recognized by a non-monotonous $\{0,1\}$-valued martingale.

Furthermore, one can find by induction values $x_{1}, x_{2}, \ldots$ such that $x_{n} \geq$ $\min \left(I_{n}\right)$ and from $x_{n}$ onwards no martingale $M_{m}$ with $m<n$ is betting on $A$. This is obviously possible for $x_{1}=\min \left(I_{1}\right)$ as the other part of the condition is void. Now, whenever $M_{1}$ bets on $A$ beyond $x_{1}$, the outcome is negative as $A$ gives highest priority to diagonalize $M_{1}$. Therefore, $M_{1}$ can bet only finitely often until the capital is used off and one can just take $x_{2}$ to be the maximum of $\min \left(I_{2}\right)$ and the last time where $M_{1}$ places a positive bet on $A$. Hence $x_{2}$ exists and from $x_{2}$ onwards, $M_{1}$ does not bet on $A$ and therefore $M_{2}$ is diagonalized with highest priority by $A$; again there are only finitely many positive bets and $x_{3}$ can be chosen as the first value after these finitely many bets and after $\min \left(I_{3}\right)$. Hence one can inductively define the $x_{n}$ and verify that $M_{n}$ never has more capital than $2^{x_{n}+1+n}$. Thus no $M_{n}$ succeeds on $A$ and $A$ is integer-valued random (with respect to $B$-recursive monotonous martingales).

We conclude with a canonical problem.

Open question 33. Do there exist other characterizations for integer-valued, finite-valued, or single-valued randoms in terms of Kolmogorov complexity or Martin-Löf statistical tests?

\section{References}

1. Laurent Bienvenu and Wolfgang Merkle. Constructive equivalence relations for computable probability measures. Annals of Pure and Applied Logic, 160:238-254, 2009.

2. Patrick Billingsley. Probability and measure. Wiley Series in Probability and Mathematical Statistics. John Wiley \& Sons Inc., New York, third edition, 1995. A Wiley-Interscience Publication. 
3. J. L. Doob. Stochastic processes. John Wiley \& Sons Inc., New York, 1953.

4. Rod Downey and Denis Hirschfeldt. Algorithmic randomness and complexity. Springer, 2010.

5. Shizuo Kakutani. On equivalence of infinite product measures. Annals of Mathematics, 49(214-224), 1948.

6. Stuart Kurtz. Randomness and genericity in the degrees of unsolvability. $\mathrm{PhD}$ dissertation, University of Illinois at Urbana, 1981.

7. Wolfgang Merkle, Joseph S. Miller, André Nies, Jan Reimann, and Frank Stephan. Kolmogorov-Loveland randomness and stochasticity. Annals of Pure and Applied Logic, 138(1-3):183-210, 2006.

8. Andrei A. Muchnik, Alexei Semenov, and Vladimir Uspensky. Mathematical metaphysics of randomness. Theoretical Computer Science, 207(2):263-317, 1998.

9. André Nies. Computability and randomness, volume 51 of Oxford Logic Guides. Oxford University Press, Oxford, 2009.

10. Sheldon M. Ross. Stochastic processes. Wiley Series in Probability and Statistics: Probability and Statistics. John Wiley \& Sons Inc., New York, second edition, 1996.

11. Claus-Peter Schnorr. Zufälligkeit und Wahrscheinlichkeit. Eine algorithmische Begründung der Wahrscheinlichkeitstheorie. Lecture Notes in Mathematics, Vol. 218. Springer-Verlag, Berlin, 1971.

12. Alexander Shen. On relations between different algorithmic definitions of randomness. Soviet Mathematics Doklady, 38:316-319, 1989.

13. Robert I. Soare. Recursively enumerable sets and degrees. Perspectives in Mathematical Logic. Springer-Verlag, Berlin, 1987. A study of computable functions and computably generated sets.

14. Vladimir Vovk. On a criterion for randomness. Soviet Mathematics Doklady, 294(6):1298-1302, 1987.

15. Yongge Wang. A separation of two randomness concepts. Information Processing Letters, 69(3):115-118, 1999. 